

\title{
On the algebraic structure of iterated integrals of quasimodular forms
}

\author{
Nils Matthes
}

\begin{abstract}
We study the algebra $\mathcal{I}^{\mathrm{QM}}$ of iterated integrals of quasimodular forms for $\mathrm{SL}_{2}(\mathbb{Z})$, which is the smallest extension of the algebra $\mathrm{QM}_{*}$ of quasimodular forms which is closed under integration. We prove that $\mathcal{I}^{\mathrm{QM}}$ is a polynomial algebra in infinitely many variables, given by Lyndon words on certain monomials in Eisenstein series. We also prove an analogous result for the $M_{*}$-subalgebra $\mathcal{I}^{M}$ of $\mathcal{I}^{\mathrm{QM}}$ of iterated integrals of modular forms.
\end{abstract}

\section{Introduction}

Quasimodular forms, a generalization of modular forms, were first introduced in [Kaneko and Zagier 1995] in a context motivated by mathematical physics. The $\mathbb{C}$-algebra $\mathrm{QM}_{*}$ of quasimodular forms for the full modular group $\mathrm{SL}_{2}(\mathbb{Z})$ can be defined, in a slightly ad hoc fashion, as the polynomial ring $\mathbb{C}\left[E_{2}, E_{4}, E_{6}\right]$, where $E_{2 k}$ denotes the normalized Eisenstein series of weight $2 k$ :

$$
E_{2 k}(\tau)=1-\frac{4 k}{B_{2 k}} \sum_{n=1}^{\infty} n^{2 k-1} \frac{q^{n}}{1-q^{n}}, \quad q=e^{2 \pi i \tau},
$$

where $B_{2 k}$ are the Bernoulli numbers. In particular, $\mathrm{QM}_{*}$ contains the algebra of modular forms $M_{*} \cong \mathbb{C}\left[E_{4}, E_{6}\right]$.

The derivative of a quasimodular form (of weight $k$ ) is again a quasimodular form (of weight $k+2$ ); this was essentially already known to Ramanujan (see [Zagier 2008, Proposition 15]). On the other hand, the integral of a quasimodular form is in general not quasimodular. For example, a primitive of $E_{2}$ would have to be of weight zero, but every quasimodular form of weight zero is constant.

The goal of this paper is to study the smallest algebra extension of $\mathrm{QM}_{*}$ which is closed under integration. For this, the idea is to iteratively adjoin primitives

MSC2010: primary 11F11; secondary $11 \mathrm{~F} 67$.

Keywords: quasimodular forms, iterated integrals. 
to $\mathrm{QM}_{*}$, which eventually leads to adjoining all (indefinite) iterated integrals

$$
I\left(f_{1}, \ldots, f_{n} ; \tau\right)=(2 \pi i)^{n} \int_{\tau \leq \tau_{1} \leq \cdots \leq \tau_{n} \leq i \infty} \cdots \int_{1} f_{1}\left(\tau_{1}\right) \cdots f_{n}\left(\tau_{n}\right) \mathrm{d} \tau_{1} \cdots \mathrm{d} \tau_{n},
$$

where $f_{1}, \ldots, f_{n}$ are quasimodular forms (a precise definition will be given in Definition 2.6). The integrals (1-1) were first studied by Manin [2006], and later by Brown [2016] and Hain [2016], in the case where all the $f_{i}$ are modular forms. ${ }^{1}$ In all of these treatments, the focus lies rather on arithmetic aspects of these iterated integrals, for example their special values at cusps of the upper half-plane. By contrast, we study them solely as holomorphic functions of $\tau$. It is also worth noting that even in the modular case, the iterated integrals we study in the present paper are slightly more general than the ones introduced in [Manin 2006; Brown 2016; Hain 2016]. For example, if $f(\tau)$ is a modular form of weight $k$, then the integral $\int_{\tau}^{i \infty} f\left(\tau_{1}\right) \tau_{1}^{n} \mathrm{~d} \tau_{1}$ is an iterated integral of modular forms in the sense of the present paper for every $n \geq 0$, while [Manin 2006; Brown 2016; Hain 2016] also require $n \leq k-2$.

Now let $\mathcal{I}^{\mathrm{QM}}$ be the $\mathrm{QM}_{*}$-algebra generated by all the integrals (1-1), which is the smallest algebra extension of $\mathrm{QM}_{*}$ closed under integration. It turns out that $\mathcal{I}^{\mathrm{QM}}$ is not finitely generated, but still has a manageable structure, which is captured by the notion of shuffle algebra (which is just the graded dual of the tensor algebra with a certain commutative multiplication, the so-called shuffle product) [Reutenauer 1993]. More precisely, let $V=\mathbb{C} \cdot E_{2} \oplus M_{*}$ be the $\mathbb{C}$-vector space spanned by all modular forms and the Eisenstein series $E_{2}$, and let $\mathbb{C}\langle V\rangle$ be the shuffle algebra on $V$. Our main result is the following.

Theorem (Theorem 4.3). The $\mathrm{QM}_{*}$-linear morphism

$$
\varphi^{\mathrm{QM}}: \mathrm{QM}_{*} \otimes_{\mathbb{C}} \mathbb{C}\langle V\rangle \rightarrow \mathcal{I}^{\mathrm{QM}}, \quad\left[f_{1}|\cdots| f_{n}\right] \mapsto I\left(f_{1}, \ldots, f_{n} ; \tau\right)
$$

is an isomorphism of $\mathrm{QM}_{*}$-algebras.

A similar result holds for the $M_{*}$-subalgebra $\mathcal{I}^{M}$ of $\mathcal{I}^{\mathrm{QM}}$ of iterated integrals of modular forms (see Theorem 4.5). ${ }^{2}$ The surjectivity of $\varphi^{\mathrm{QM}}$ can be reduced to the fact that every quasimodular form can be written uniquely as a polynomial in $n$-th derivatives of modular forms and the Eisenstein series $E_{2}$; see [Zagier 2008, Proposition 20]. The proof of injectivity is more elaborate and amounts to showing that iterated integrals of modular forms and the Eisenstein series $E_{2}$ are linearly

\footnotetext{
${ }^{1}$ More precisely, Manin only defined iterated integrals of cusp forms, and the extension to all modular forms is due to Brown.

${ }^{2}$ After this paper was submitted for publication, the author learned that, in the case of iterated integrals of modular forms, a very similar result has also been proved by Brown [2017, Proposition 4.4] using a slightly different method.
} 
independent over $\mathrm{QM}_{*}$. It extends results of [Lochak et al. 2017], which dealt with iterated integrals of Eisenstein series. In both cases, the key is to use a general result on linear independence of iterated integrals [Deneufchâtel et al. 2011]. It would be interesting to prove similar results for quasimodular forms for congruence subgroups.

The Milnor-Moore theorem [Milnor and Moore 1965] states that if $k$ has characteristic zero, then $k\langle V\rangle$ is isomorphic to a polynomial algebra (usually in infinitely many variables). Fixing a (totally ordered) basis $\mathcal{B}$ of $V$, Radford [1979] has given explicit generators of $k\langle V\rangle$ in terms of Lyndon words on $\mathcal{B}$ (see Section 4). Using this, we get the following theorem.

Theorem (Theorem 4.9). Let $\mathcal{B}$ be a basis of $\mathbb{C} \cdot E_{2} \oplus M_{*}$. We have a natural isomorphism

$$
\mathcal{I}^{\mathrm{QM}} \cong \mathrm{QM}_{*}\left[\operatorname{Lyn}\left(\mathcal{B}^{*}\right)\right] \text {, }
$$

where the right-hand side is the polynomial $\mathrm{QM}_{*}$-algebra on the set $\operatorname{Lyn}\left(\mathcal{B}^{*}\right)$ of Lyndon words of $\mathcal{B}$.

Again, a similar result holds for $\mathcal{I}^{M}$. Since $\mathrm{QM}_{*}$ has an explicit basis given by monomials in the Eisenstein series $E_{2}, E_{4}$ and $E_{6}$, the isomorphism (1-2) can be made completely explicit, and may be viewed as an analog of the isomorphism $\mathrm{QM}_{*} \cong \mathbb{C}\left[E_{2}, E_{4}, E_{6}\right]$ [Kaneko and Zagier 1995].

Finally, we note that classically, integrals of modular forms play an important role in Eichler-Shimura theory, where they give rise to group-cocycles (say for $\mathrm{SL}_{2}(\mathbb{Z})$ or more generally for some congruence subgroup thereof) with values in homogeneous polynomials. This has been generalized by Manin [2006], and later by Brown [2016] and Hain [2016], who attach certain nonabelian cocycles to iterated integrals of modular forms. Although it is not the main focus of this article, in the Appendix we show how one can attach cocycles to quasimodular forms (for $\mathrm{SL}_{2}(\mathbb{Z})$ ), partly since we found no mention of this in the literature. On the other hand, we leave the definition and study of cocycles attached to iterated integrals of quasimodular forms for future investigation.

The plan of the paper is as follows. In Section 2, we collect the necessary background on quasimodular forms and their iterated integrals. In Section 3, we prove a linear independence result for iterated integrals of quasimodular forms. This result is then put to use in Section 4, where the main results are proved. In the Appendix, we discuss the above-mentioned generalization of the classical Eichler-Shimura theory to quasimodular forms for $\mathrm{SL}_{2}(\mathbb{Z})$.

\section{Preliminaries}

Throughout the paper, all modular and quasimodular forms will be for $\mathrm{SL}_{2}(\mathbb{Z})$. We fix some notation. Let $\mathfrak{H}=\{z \in \mathbb{C} \mid \operatorname{Im}(z)>0\}$ be the upper half-plane with canonical coordinate $\tau$. For every $k \in \mathbb{Z}$, we have a group action of $\mathrm{SL}_{2}(\mathbb{Z})$ on the set of all 
functions $f: \mathfrak{H} \rightarrow \mathbb{C}$ (not necessarily holomorphic), defined by $\left.(\gamma, f) \mapsto f\right|_{k} \gamma$, where

$$
\left(\left.f\right|_{k} \gamma\right)(\tau):=(c \tau+d)^{-k} f\left(\frac{a \tau+b}{c \tau+d}\right) \text {. }
$$

For fixed $\tau \in \mathfrak{H}$, we also define a map $X: \mathrm{SL}_{2}(\mathbb{Z}) \rightarrow \mathbb{C}$ by $X(\gamma)=\frac{1}{2 \pi i} \frac{c}{c \tau+d}$. Note that $X$ has infinite, and thus Zariski dense, image.

Recap of modular forms. Denote by $M_{k}$ the space of modular forms of weight $k \in \mathbb{Z}$. By definition, these are the holomorphic functions $f: \mathfrak{H} \rightarrow \mathbb{C}$, which satisfy $\left.f\right|_{k} \gamma=f$ for all $\gamma \in \mathrm{SL}_{2}(\mathbb{Z})$, and which are "holomorphic at the cusp". The latter condition means that in the Fourier expansion $f(\tau)=\sum_{n \in \mathbb{Z}} a_{n} q^{n}$ (which exists since for $\gamma=\left(\begin{array}{ll}1 & 1 \\ 0 & 1\end{array}\right) \in \mathrm{SL}_{2}(\mathbb{Z})$, the condition $\left.f\right|_{k} \gamma=f$ is just $f(\tau+1)=f(\tau)$ for all $\tau), a_{n}=0$ for all $n<0$. Examples of modular forms include the Eisenstein series

$$
E_{2 k}(\tau)=1-\frac{4 k}{B_{2 k}} \sum_{n=1}^{\infty} n^{2 k-1} \frac{q^{n}}{1-q^{n}}=1-\frac{4 k}{B_{2 k}} \sum_{n=1}^{\infty}\left(\sum_{d \mid n} d^{2 k-1}\right) q^{n},
$$

which is a modular form of weight $2 k$, for $k \geq 2$ (the $B_{2 k}$ are Bernoulli numbers). The $\mathbb{C}$-vector space of all modular forms $M_{*}$ is a graded (for the weight) $\mathbb{C}$-algebra $M_{*}=\bigoplus_{k \in \mathbb{Z}} M_{k}$, which is well-known to be isomorphic to the polynomial algebra $\mathbb{C}\left[E_{4}, E_{6}\right]$. Proofs of all these facts and much more on modular forms can be found, for example, in [Zagier 2008].

Quasimodular forms. Quasimodular forms are a generalization of modular forms which was first introduced in [Kaneko and Zagier 1995]; see also [Bloch and Okounkov 2000, §3; Zagier 2008, §5.3]. The definition we give here is due to W. Nahm ${ }^{3}$ and is also used for example in [Martin and Royer 2005].

Definition 2.1. Let $k, p \in \mathbb{Z}$ with $p \geq 0$. A quasimodular form of weight $k$ and depth $\leq p$ is a function $f: \mathfrak{H} \rightarrow \mathbb{C}$ with the following property: there exist holomorphic functions $f_{r}: \mathfrak{H} \rightarrow \mathbb{C}$, for $0 \leq r \leq p$, which have Fourier expansions $\sum_{n=0}^{\infty} a_{n} q^{n}$ such that

$$
\left(\left.f\right|_{k} \gamma\right)(\tau)=\sum_{r=0}^{p} f_{r}(\tau) X(\gamma)^{r}, \quad \text { for all } \gamma \in \mathrm{SL}_{2}(\mathbb{Z}) \text {. }
$$

We denote by $\mathrm{QM}_{k}^{\leq p}$ the $\mathbb{C}$-vector space of quasimodular forms of weight $k$ and depth $\leq p$, and set

$$
\mathrm{QM}_{k}:=\bigcup_{p \geq 0} \mathrm{QM}_{k}^{\leq p}, \quad \mathrm{QM}_{*}:=\bigoplus_{k \in \mathbb{Z}} \mathrm{QM}_{k} .
$$

${ }^{3}$ See [Zagier 2008, §5.3]. 
Remark 2.2. (i) It is clear from the definition that, if $f_{1} \in \mathrm{QM}_{k_{1}}^{\leq p_{1}}, f_{2} \in \mathrm{QM}_{k_{2}}^{\leq p_{2}}$, then $f_{1} f_{2} \in \mathrm{QM}_{k_{1}+k_{2}}^{\leq p_{1}+p_{2}}$. In other words, $\mathrm{QM}_{*}$ is a graded (for the weight) and filtered (for the depth) $\mathbb{C}$-algebra.

(ii) Using the fact that $X$ is Zariski dense, it is easy to see that the functions $f_{r}(\tau)$ are uniquely determined by $f(\tau)$. Also, applying (2-1) with $\gamma=\left(\begin{array}{ll}1 & 0 \\ 0 & 1\end{array}\right)$, we see that $f_{0}(\tau)=f(\tau)$. In particular, every quasimodular form is holomorphic on $\mathfrak{H}$ and at the cusp.

Every modular form is a quasimodular form of depth zero; more precisely, $M_{k}=\mathrm{QM}_{k}^{\leq 0}$. An example of a quasimodular form which is not modular is the Eisenstein series of weight two $E_{2}(\tau)=1-24 \sum_{n=1}^{\infty} n \frac{q^{n}}{1-q^{n}}$, which transforms as

$$
\left(\left.E_{2}\right|_{2} \gamma\right)(\tau)=E_{2}(\tau)+12 X(\gamma)=E_{2}(\tau)-\frac{6 i}{\pi} \frac{c}{c \tau+d}
$$

for all $\gamma \in \mathrm{SL}_{2}(\mathbb{Z})$. In particular, $E_{2} \in \mathrm{QM}_{2}^{\leq 1} \backslash M_{2}$.

The following proposition recalls basic properties of $\mathrm{QM}_{*}$ that will be of use later.

Proposition 2.3. (i) The $\mathbb{C}$-algebra $\mathrm{QM}_{*}$ is closed under the differential operator $D:=\frac{1}{2 \pi i} \frac{d}{d \tau}=q \frac{d}{d q}$. More precisely, for $f$ quasimodular of weight $k$ and depth $\leq p$, we have

$$
\left(\left.D(f)\right|_{k+2} \gamma\right)(\tau)=\sum_{r=0}^{p+1}\left(D\left(f_{r}\right)(\tau)+(k-r+1) f_{r-1}(\tau)\right) X(\gamma)^{r} .
$$

In particular, $D\left(\mathrm{QM}_{k}^{\leq p}\right) \subset \mathrm{QM}_{k+2}^{\leq p+1}$ for all $k, p \in \mathbb{Z}$.

(ii) We have

$$
\mathrm{QM}_{k}= \begin{cases}\{0\} & \text { if } k<0, \\ \mathbb{C} \cdot E_{2} & \text { if } k=2, \\ D\left(\mathrm{QM}_{k-2}\right) \oplus M_{k} & \text { else. }\end{cases}
$$

In particular, $\mathrm{QM}_{*}=\mathbb{C} \cdot E_{2} \oplus D\left(\mathrm{QM}_{*}\right) \oplus M_{*}$, and

$$
\mathrm{QM}_{*} \cong \mathbb{C}\left[E_{2}, E_{4}, E_{6}\right]
$$

as graded $\mathbb{C}$-algebras.

Proof. For (i), simply apply $D$ to both sides of (2-1). The first equality in (ii) follows from [Zagier 2008, Proposition 20(iii)], and the isomorphism $\mathrm{QM}_{*} \cong \mathbb{C}\left[E_{2}, E_{4}, E_{6}\right]$ is essentially a consequence of this, but can also be proved independently (see [Bloch and Okounkov 2000, Proposition 3.5(ii)]).

Remark 2.4. Relaxing the condition in the definition of quasimodular forms that every $f_{r}$ be a holomorphic function, one can define the notion of weakly quasimodular form of weight $k$ and depth $\leq p$ as a meromorphic function $f: \mathfrak{H} \rightarrow \mathbb{C}$ satisfying (2-1), but where the functions $f_{r}(\tau)$ are only required to be meromorphic on $\mathfrak{H}$ and have Fourier series of the form $\sum_{n=-M}^{\infty} a_{n} q^{n}$ ( $f_{r}$ is "meromorphic 
at the cusp"). As in the case of quasimodular forms, one shows easily that the functions $f_{r}(\tau)$ are uniquely determined by $f(\tau)$ (see Remark 2.2). Moreover, Proposition 2.3(i) generalizes straightforwardly to weakly quasimodular forms.

We end this subsection with a short lemma, for which we couldn't find a suitable reference. Denote by $\Delta=\frac{1}{1728}\left(E_{4}^{3}-E_{6}^{2}\right)$ Ramanujan's cusp form of weight 12 .

Lemma 2.5. Let $g \in \mathrm{QM}_{*} \backslash\{0\}$ and $\alpha \in \mathbb{C}$ be such that

$$
D(g)=\left(\alpha E_{2}\right) \cdot g .
$$

Then $\alpha$ is a nonnegative integer, and $g=\beta \Delta^{\alpha}$ for some $\beta \in \mathbb{C} \backslash\{0\}$.

Proof. Let $g=\sum_{n=0}^{\infty} a_{n} q^{n}$, so that $D(g)=\sum_{n=0}^{\infty} n a_{n} q^{n}$. Comparing coefficients on both sides of (2-3) yields that $\alpha$ equals the smallest integer $m \geq 0$ such that $a_{m} \neq 0$. On the other hand, $D(\Delta) / \Delta=E_{2}$ [Zagier 2008, proof of Proposition 7], and from the chain rule, $D\left(\Delta^{\alpha}\right) / \Delta^{\alpha}=\alpha E_{2}$, which gives the result.

Iterated integrals on the upper half-plane. Iterated integrals of modular forms were first considered by Manin [2006] (for cusp forms), and later by Brown [2016] (in general). They are generalizations of the classical Eichler integrals

$$
\int_{\tau}^{i \infty} f(z) z^{m} \mathrm{~d} z, \quad m=0, \ldots, k-2,
$$

where $f$ is a cusp form of weight $k$ [Eichler 1957; Lang 1976]. Extending (2-4) to a general modular form poses the problem of logarithmic divergences, which arise from the constant term in the Fourier series of $f$. A procedure for regularizing such integrals is described in [Brown 2016], and we borrow it to define iterated integrals of quasimodular forms. Since it is perhaps not so well-known, we give some details for the convenience of the reader.

Let $W \subset \mathcal{O}(\mathfrak{H})$ be the $\mathbb{C}$-subalgebra of holomorphic functions $f: \mathfrak{H} \rightarrow \mathbb{C}$, which have an everywhere convergent Fourier series $f(\tau)=\sum_{n=0}^{\infty} a_{n} q^{n}$ with $q=e^{2 \pi i \tau}$. Note that $\mathrm{QM}_{*} \subset W$. For $f(\tau) \in W$, let $f^{\infty}=a_{0}$, and $f^{0}(\tau)=f(\tau)-f^{\infty}=$ $\sum_{n=1}^{\infty} a_{n} q^{n}$. Let $\mathbb{C}\langle W\rangle$ (sometimes denoted by $T^{c}(W)$ ) be the shuffle algebra [Reutenauer 1993], i.e., the graded dual of the tensor algebra $T(W)=\bigoplus_{k \geq 0} W^{\otimes n}$ on $W$, where the grading is by the length of tensors. Elements of $\left(W^{\otimes n}\right)^{\vee}$ will be written using bar notation $\left[f_{1}\left|f_{2}\right| \cdots \mid f_{n}\right]$, and a general element of $\mathbb{C}\langle W\rangle$ is a $\mathbb{C}$-linear combination of those. The product on $\mathbb{C}\langle W\rangle$ is the shuffle product $\sqcup$, which is defined on the basic elements by

$$
\left[f_{1}|\cdots| f_{r}\right] \sqcup\left[f_{r+1}|\cdots| f_{r+s}\right]=\sum_{\sigma \in \Sigma_{r, s}}\left[f_{\sigma(1)}|\cdots| f_{\sigma(r+s)}\right],
$$

where $\Sigma_{r, s}$ denotes the set of all the permutations on the set $\{1, \ldots, r+s\}$ such that $\sigma^{-1}(1)<\cdots<\sigma^{-1}(r)$ and $\sigma^{-1}(r+1)<\cdots<\sigma^{-1}(r+s)$. 
Define a $\mathbb{C}$-linear map $R: \mathbb{C}\langle W\rangle \rightarrow \mathbb{C}\langle W\rangle$ by the formula

$$
R\left[f_{1}|\cdots| f_{n}\right]=\sum_{i=0}^{n}(-1)^{n-i}\left[f_{1}|\cdots| f_{i}\right] \uplus\left[f_{n}^{\infty}|\cdots| f_{i+1}^{\infty}\right] .
$$

Following [Brown 2016, §4], we make the following definition.

Definition 2.6. For $f_{1}, \ldots, f_{n} \in W$, define their regularized iterated integral $I\left(f_{1}, \ldots, f_{n} ; \tau\right)$

where

$$
:=(2 \pi i)^{n} \sum_{i=0}^{n}(-1)^{n-i} \int_{\tau}^{i \infty} R\left[f_{1}|\cdots| f_{i}\right] \int_{0}^{\tau}\left[f_{n}^{\infty}|\cdots| f_{i+1}^{\infty}\right]
$$

$$
\int_{a}^{b}\left[f_{1}|\cdots| f_{n}\right]:=\int_{0 \leq t_{1} \leq \cdots \leq t_{n} \leq 1}\left(\gamma_{a}^{b}\right)^{*}\left(f_{1}\left(\tau_{1}\right) \mathrm{d} \tau_{1}\right) \cdots\left(\gamma_{a}^{b}\right)^{*}\left(f_{n}\left(\tau_{n}\right) \mathrm{d} \tau_{n}\right)
$$

denotes the usual iterated integral along the straight line path $\gamma_{a}^{b}$ from $a$ to $b$.

Remark 2.7. Using the change of variables $\tau \mapsto q=e^{2 \pi i \tau}$, it is easy to see that $I\left(f_{1}, \ldots, f_{n} ; \tau\right) \in W[\log (q)]$, where $\log (q):=2 \pi i \tau$. By the same token, if all of the $f_{i}$ have rational Fourier coefficients, then $I\left(f_{1}, \ldots, f_{n} ; \tau\right)$ will also have rational coefficients, as a series in $q$ and $\log (q)$.

Proposition 2.8. The functions $I\left(f_{1}, \ldots, f_{n} ; \tau\right)$ satisfy the following properties.

(i) The product of any two of them is given by the shuffle product

$$
I\left(f_{1}, \ldots, f_{r} ; \tau\right) I\left(f_{r+1}, \ldots, f_{r+s} ; \tau\right)=\sum_{\sigma \in \Sigma_{r, s}} I\left(f_{\sigma(1)}, \ldots, f_{\sigma(r+s)} ; \tau\right) .
$$

(ii) They satisfy the differential equation

$$
\left.\frac{1}{2 \pi i} \frac{d}{d \tau}\right|_{\tau=\tau_{0}} I\left(f_{1}, \ldots, f_{n} ; \tau\right)=-f_{1}\left(\tau_{0}\right) I\left(f_{2}, \ldots, f_{n} ; \tau_{0}\right) .
$$

(iii) We have the integration by parts formulas

$$
\begin{aligned}
& I\left(f_{1}, \ldots, f_{i}, D(g), f_{i+1}, \ldots, f_{n} ; \tau\right) \\
& \quad=I\left(f_{1}, \ldots, f_{i}, g f_{i+1}, \ldots, f_{n} ; \tau\right)-I\left(f_{1}, \ldots, f_{i} g, f_{i+1}, \ldots, f_{n} ; \tau\right),
\end{aligned}
$$

as well as

$$
I\left(D(g), f_{2}, \ldots, f_{n} ; \tau\right)=I\left(g f_{2}, f_{3}, \ldots, f_{n} ; \tau\right)-g(\tau) I\left(f_{2}, \ldots, f_{n} ; \tau\right),
$$

and

$$
I\left(f_{1}, \ldots, f_{n-1}, D(g) ; \tau\right)=g(i \infty) I\left(f_{1}, \ldots, f_{n-1} ; \tau\right)-I\left(f_{1}, \ldots, f_{n-1} g ; \tau\right) .
$$

Proof. Using the definition (2-6), all of these follow from the analogous properties for usual iterated integrals; see, e.g., [Hain 1987]. 
A criterion for linear independence of iterated integrals. Let $\operatorname{Frac}(W)$ be the field of fractions of the $\mathbb{C}$-algebra $W$ introduced in the last subsection. By the quotient rule, it is easy to see that $\operatorname{Frac}(W)$ is closed under $D=\frac{1}{2 \pi i} \frac{d}{d \tau}$.

The following theorem is a special case of the main result of [Deneufchâtel et al. 2011].

Theorem 2.9. Let $\mathcal{F}=\left(f_{i}\right)_{i \in I}$ be a family of elements of $W$, and let $\mathcal{C} \subset \operatorname{Frac}(W)$ be a subfield which is closed under $D$ and contains $\mathcal{F}$. The following are equivalent:

(i) The family of iterated integrals $\left(I\left(f_{1}, \ldots, f_{n} ; \tau\right) \mid f_{i} \in I, n \geq 0\right)$ is linearly independent over $\mathcal{C}$.

(ii) The family $\mathcal{F}$ is linearly independent over $\mathbb{C}$, and we have

$$
D(\mathcal{C}) \cap \operatorname{Span}_{\mathbb{C}}(\mathcal{F})=\{0\} .
$$

Proof. This is the special case of Theorem 2.1 in [Deneufchâtel et al. 2011], with the notation $k=\mathbb{C},(\mathcal{A}, \mathrm{d})=(\operatorname{Frac}(\mathcal{O}(\mathfrak{H})), D), X=\left\{A_{f_{i}} \mid f_{i} \in \mathcal{F}\right\}, M=-\sum_{i \in I} f_{i} A_{f_{i}}$ and $S=\sum_{n \geq 0} \sum_{f_{i_{1}}, \ldots, f_{i_{n}} \in S} I\left(f_{1}, \ldots, f_{n} ; \tau\right) \cdot A_{f_{1}} \cdots A_{f_{n}}$. Note that it follows from (2-8) that

$$
D(S)=M \cdot S,
$$

as required in [loc. cit.].

Remark 2.10. Variants of Theorem 2.9 have been known before; see [Brown 2009, Lemma 3.6].

\section{Linear independence of iterated integrals of quasimodular forms}

In this section, we apply Theorem 2.9 to deduce linear independence of a large family of iterated integrals of quasimodular forms. More precisely, our main result is the following theorem.

Theorem 3.1. Let $\mathcal{B}$ be a $\mathbb{C}$-linearly independent family of elements of $\mathbb{C} \cdot E_{2} \oplus M_{*}$. Then the family of iterated integrals

$$
\left(I\left(f_{1}, \ldots, f_{n} ; \tau\right) \mid f_{i} \in \mathcal{B}\right)
$$

is linearly independent over $\operatorname{Frac}\left(\mathrm{QM}_{*}\right) \cong \mathbb{C}\left(E_{2}, E_{4}, E_{6}\right)$.

Two auxiliary lemmas. For the proof of Theorem 3.1, we need two lemmas.

Lemma 3.2. Let $f, g \in \mathbb{C}\left[E_{2}, E_{4}, E_{6}\right]$ be such that $g \neq 0$ and such that $f$ and $g$ are coprime. Assume that $D(f / g) \in \mathbb{C}\left[E_{2}, E_{4}, E_{6}\right]$. Then $g=\beta \Delta^{\alpha}$ for some $\alpha \in \mathbb{Z}_{\geq 0}$ and some $\beta \in \mathbb{C} \backslash\{0\}$, where $\Delta:=\frac{1}{1728}\left(E_{4}^{3}-E_{6}^{2}\right)$ is Ramanujan's cusp form of weight 12 . 
Proof. By the quotient rule, we have

$$
D\left(\frac{f}{g}\right)=\frac{D(f) g-f D(g)}{g^{2}}=\frac{D(f)-f D(g) / g}{g} .
$$

The left-hand side is contained in $\mathbb{C}\left[E_{2}, E_{4}, E_{6}\right]$ by assumption, and since also $D(f)$ and $g$ are in $\mathbb{C}\left[E_{2}, E_{4}, E_{6}\right]$, we have $f D(g) / g \in \mathbb{C}\left[E_{2}, E_{4}, E_{6}\right]$. But then, as $f$ and $g$ have no common factor, $g$ must divide $D(g)$, i.e., there exists $h \in \mathbb{C}\left[E_{2}, E_{4}, E_{6}\right]$ such that

$$
D(g)=g h .
$$

Since $D: \mathrm{QM}_{*} \rightarrow \mathrm{QM}_{*}$ is homogeneous of weight 2 (see Proposition 2.3(i)), we have $h \in \mathrm{QM}_{2}$, i.e., $h=\alpha E_{2}$ with $\alpha \in \mathbb{C}$. In other words, $g$ solves the differential equation $D(g)=\left(\alpha E_{2}\right) \cdot g$. But by Lemma 2.5, $\alpha$ must be a nonnegative integer and $g=\beta \Delta^{\alpha}$ for some $\beta \in \mathbb{C} \backslash\{0\}$.

Lemma 3.3. Let $f$ be a weakly quasimodular form such that its derivative $D(f)$ is a quasimodular form. Then $f$ is a quasimodular form.

Proof. It is no loss of generality to assume that $f$ is of weight $k \in \mathbb{Z}$ and depth $\leq p$, where $p \geq 0$. By the definition of weakly quasimodular forms (see also Remark 2.2), there exist uniquely determined meromorphic functions $f_{r}(\tau)$, for $0 \leq r \leq p$, such that

$$
\left(\left.f\right|_{k} \gamma\right)(\tau)=\sum_{r=0}^{p} f_{r}(\tau) X(\gamma)^{r}
$$

for all $\gamma \in \mathrm{SL}_{2}(\mathbb{Z})$. Therefore, we only need to show that every $f_{r}(\tau)$ is holomorphic, including at the cusp.

To this end, by Proposition 2.3(i), we know that

$$
\left(\left.D(f)\right|_{k+2} \gamma\right)(\tau)=\sum_{r=0}^{p+1}\left(D\left(f_{r}\right)(\tau)+(k-r+1) f_{r-1}(\tau)\right) X(\gamma)^{r},
$$

and since $D(f)$ is a quasimodular form by assumption, every coefficient of (3-1) is holomorphic, including at the cusp.

The constant term, with respect to $X(\gamma)$, in (3-1) equals $D\left(f_{0}\right)(\tau)$, which is holomorphic by assumption. But a meromorphic function whose derivative is holomorphic everywhere is itself holomorphic everywhere. An easy induction argument, using the fact that the coefficients of (3-1) are holomorphic, now shows that in fact every $f_{r}(\tau)$ is holomorphic.

Proof of Theorem 3.1. We use the criterion of Theorem 2.9 in the case where $\mathcal{C}=\operatorname{Frac}\left(\mathrm{QM}_{*}\right)$ and $\mathcal{F}=\mathcal{B}$. Since $\mathcal{B}$ is linearly independent over $\mathbb{C}$ by assumption, it is enough to prove that if $h \in \operatorname{Frac}\left(\mathrm{QM}_{*}\right)$ then

$$
D(h)=\sum_{f \in \mathcal{B}} \alpha_{f} f \text { and } \alpha_{f} \in \mathbb{C} \Rightarrow \alpha_{f}=0 \text {, for all } f \in \mathcal{B} .
$$


Also, since $\mathcal{B}$ spans a subspace of $\mathbb{C} \cdot E_{2} \oplus M_{*}$, it clearly suffices to prove that $D(h) \in \mathbb{C} \cdot E_{2} \oplus M_{*}$ implies that $D(h)=0$, or equivalently, that $h$ is constant. Thus, the following proposition completes the proof of Theorem 3.1.

Proposition 3.4. Suppose that $h \in \operatorname{Frac}\left(\mathrm{QM}_{*}\right) \cong \mathbb{C}\left(E_{2}, E_{4}, E_{6}\right)$ is such that $D(h) \in \mathbb{C} \cdot E_{2} \oplus M_{*}$. Then $h$ is constant.

Proof. Write $h=f / g$ with $f, g \in \mathbb{C}\left[E_{2}, E_{4}, E_{6}\right]$ such that $g \neq 0$ and $f$ and $g$ are coprime. Writing $f$ as a $\mathbb{C}$-linear combination of its homogeneous components, it is enough to show the proposition for $f$ homogeneous of weight $k_{f}$.

First, we know from Lemma 3.2 that $g=\beta \Delta^{\alpha}$ for some $\alpha \in \mathbb{Z}_{\geq 0}$ and $\beta \in \mathbb{C} \backslash\{0\}$, where $\Delta$ is Ramanujan's cusp form of weight 12. In particular, $g$ is a cusp form of weight $k_{g}=12 \alpha$.

Since $f$ is quasimodular of weight $k_{f}$ and depth $\leq p$, there exist holomorphic (including at the cusp) functions $f_{r}(\tau)$, for $0 \leq r \leq p$, such that

$$
\left(\left.f\right|_{k_{f}} \gamma\right)(\tau)=\sum_{r=0}^{p} f_{r}(\tau) X(\gamma)^{r}
$$

for all $\gamma \in \mathrm{SL}_{2}(\mathbb{Z})$. Setting $h_{r}(\tau):=\frac{f_{r}}{g}(\tau)$, we also have, for $k:=k_{f}-k_{g}$,

$$
\left(\left.h\right|_{k} \gamma\right)(\tau)=\sum_{r=0}^{p} h_{r}(\tau) X(\gamma)^{r} .
$$

Moreover, the functions $h_{r}(\tau)$ are meromorphic; thus, $h$ is a weakly quasimodular form (of weight $k$ and depth $\leq p$ ). By assumption, $D(h)$ is a quasimodular form (necessarily of weight $k+2$ and depth $\leq p+1$ ), and using Lemma 3.3, this implies that $h \in \mathrm{QM}_{k}^{\leq p}$. Therefore, every $h_{r}(\tau)$ is holomorphic, including at the cusp.

Summarizing, we have seen that $h \in \operatorname{Frac}\left(\mathrm{QM}_{*}\right)$ such that $D(h) \in \mathrm{QM}_{*}$ implies that $h \in \mathrm{QM}_{*}$. But we even have $D(h) \in \mathbb{C} \cdot E_{2} \oplus M_{*}$ by assumption, and therefore Proposition 2.3(ii) now implies that $h$ is constant, as was to be shown.

\section{Iterated integrals of quasimodular forms and shuffle algebras}

We describe the $\mathrm{QM}_{*}$-algebra of iterated integrals of quasimodular forms, which is the smallest algebra which contains $\mathrm{QM}_{*}$ and is closed under integration. Using the results of the last section, we show that it is canonically isomorphic to an explicit shuffle algebra. A similar result holds for the $M_{*}$-subalgebra of iterated integrals of modular forms.

\section{The algebra of iterated integrals of quasimodular forms.}

Definition 4.1. Define $\mathcal{I}^{\mathrm{QM}}$ to be the $\mathrm{QM}_{*}$-module generated by all iterated integrals of quasimodular forms: 


$$
\mathcal{I}^{\mathrm{QM}}=\operatorname{Span}_{\mathrm{QM}_{*}}\left\{I\left(f_{1}, \ldots, f_{n} ; \tau\right) \mid f_{i} \in \mathrm{QM}_{*}\right\} .
$$

We also denote by $\mathcal{I}_{n}^{\mathrm{QM}}$ the $\mathrm{QM}_{*}$-linear submodule, which is spanned by all of the $I\left(f_{1}, \ldots, f_{r} ; \tau\right)$ with $r \leq n$.

The subspaces $\mathcal{I}_{n}^{\mathrm{QM}}$ define an ascending filtration $\mathcal{I}_{\bullet}^{\mathrm{QM}}$ on $\mathcal{I}^{\mathrm{QM}}$, called the length filtration (in analogy with the length filtration on iterated integrals [Hain 1987]). It follows from (2-7) that $\mathcal{I}^{\mathrm{QM}}$ is a filtered $\mathrm{QM}_{*}$-algebra. However, the length is not a grading, as shown by the next result.

Proposition 4.2. Let $f_{1}, \ldots, f_{n}$ be quasimodular forms. Then

$$
I\left(f_{1}, \ldots, f_{i-1}, D\left(f_{i}\right), f_{i+1}, \ldots, f_{n} ; \tau\right) \in \mathcal{I}_{n-1}^{\mathrm{QM}} .
$$

Proof. This follows immediately from the integration by parts formula (2-9).

$\mathcal{I}^{\mathrm{QM}}$ as a shuffle algebra. We let $V$ be the $\mathbb{C}$-vector space $\mathbb{C} \cdot E_{2} \oplus M_{*}$, and denote by $\mathbb{C}\langle V\rangle$ the shuffle algebra on $V$ (see Section 2). Recall that this is the graded dual of the tensor algebra $T(V)$, whose grading is given by the length of tensors. Elements of $\mathbb{C}\langle V\rangle$ are $\mathbb{C}$-linear combination of the basic elements $\left[f_{1}|\cdots| f_{n}\right]$, and the product on $\mathbb{C}\langle V\rangle$ is the shuffle product (2-5).

The following theorem is the main result of this paper.

Theorem 4.3. The $\mathrm{QM}_{*}$-linear map

$$
\varphi^{\mathrm{QM}}: \mathrm{QM}_{*} \otimes \mathbb{C} \mathbb{C}\langle V\rangle \rightarrow \mathcal{I}^{\mathrm{QM}}, \quad\left[f_{1}|\cdots| f_{n}\right] \mapsto I\left(f_{1}, \ldots, f_{n} ; \tau\right)
$$

is an isomorphism of $\mathrm{QM}_{*}$-algebras.

Proof. Let $\mathcal{B}$ be a basis of $V$, so that the family $\left(\left[f_{1}|\cdots| f_{n}\right] \mid f_{i} \in \mathcal{B}\right)$ is a basis of $\mathbb{C}\langle V\rangle$. The injectivity of $\varphi^{\mathrm{QM}}$ follows from the $\operatorname{Frac}\left(\mathrm{QM}_{*}\right)$-linear independence of the family

$$
\mathcal{F}=\left(I\left(f_{1}, \ldots, f_{n} ; \tau\right) \mid f_{i} \in \mathcal{B}\right),
$$

which is a consequence of Theorem 3.1.

To obtain the surjectivity, we need to prove that the family (4-2) generates $\mathcal{I}^{\mathrm{QM}}$. To this end, we prove inductively that for every $n \geq 0$, we have $\mathcal{I}_{n}^{\mathrm{QM}} \subset \operatorname{Span}_{\mathrm{QM}_{*}} \mathcal{F}$. The case $n=0$ is trivial. Now let $n \geq 1$ and assume that for every $r \leq n-1$, we have $\mathcal{I}_{r}^{\mathrm{QM}} \subset \operatorname{Span}_{\mathrm{QM}_{*}} \mathcal{F}$. Given quasimodular forms $f_{1}, \ldots, f_{n}$, we can write $f_{i}=g_{i}+D\left(h_{i}\right)$, where $g_{i} \in \mathbb{C} \cdot E_{2} \oplus M_{*}$ and $h_{i} \in D\left(\mathrm{QM}_{*}\right)$ by Proposition 2.3(ii). Then by linearity,

$$
\begin{aligned}
& I\left(f_{1}, \ldots, f_{n} ; \tau\right)=I\left(g_{1}, \ldots, g_{n} ; \tau\right) \\
& \quad+\sum_{i=1}^{n} I\left(g_{1}, \ldots, g_{i-1}, D\left(h_{i}\right), g_{i+1}, \ldots, g_{n}\right)+\cdots,
\end{aligned}
$$


where the $\cdots$ above signifies iterated integrals which have at least two $D\left(h_{i}\right)$ as integrands. The first term on the right is contained in $\operatorname{Span}_{\mathrm{QM}_{*}} \mathcal{F}$, since $g_{i} \in \mathbb{C} \cdot E_{2} \oplus M_{*}$ for every $i$ and $\mathcal{B}$ is a basis. On the other hand, all other terms in the sum (4-3) are iterated integrals which contain at least one $D\left(h_{i}\right)$. By Proposition 4.2, it thus follows that

$$
I\left(f_{1}, \ldots, f_{n} ; \tau\right) \equiv I\left(g_{1}, \ldots, g_{n} ; \tau\right) \quad \bmod \mathcal{I}_{n-1}^{\mathrm{QM}},
$$

and we conclude using the induction hypothesis. Finally, it is clear that $\varphi^{\mathrm{QM}}$ is a homomorphism of algebras, since both sides of (4-1) are endowed with the shuffle product.

The algebra of iterated integrals of modular forms. In this section, we study the subalgebra $\mathcal{I}^{M}$ of $\mathcal{I}^{\mathrm{QM}}$, generated by iterated integrals of modular forms.

Definition 4.4. Define $\mathcal{I}^{M}$ to be the $M_{*}$-module generated by all iterated integrals of modular forms:

$$
\mathcal{I}^{M}=\operatorname{Span}_{M_{*}}\left\{I\left(f_{1}, \ldots, f_{n} ; \tau\right) \mid f_{i} \in M_{*}\right\} .
$$

As in the case of $\mathcal{I}^{\mathrm{QM}}$, the length of iterated integrals defines the length filtration $\mathcal{I}_{\bullet}^{M}$ on $\mathcal{I}^{M}$, and $\mathcal{I}^{M}$ is a filtered $M_{*}$-subalgebra of $\mathcal{I}^{\mathrm{QM}}$. We let $\mathbb{C}\left\langle M_{*}\right\rangle$ be the shuffle algebra on the $\mathbb{C}$-vector space $M_{*}$.

Theorem 4.5. The $M_{*}$-linear map

$$
\varphi^{M}: M_{*} \otimes_{\mathbb{C}} \mathbb{C}\left\langle M_{*}\right\rangle \rightarrow \mathcal{I}^{M}, \quad\left[f_{1}|\cdots| f_{n}\right] \mapsto I\left(f_{1}, \ldots, f_{n} ; \tau\right)
$$

is an isomorphism of $M_{*}$-algebras.

Proof. The morphism $\varphi^{M}$ is surjective by definition. It is also injective, since for a basis $\mathcal{B}_{M}$ of $M_{*}$, the iterated integrals $I\left(f_{1}, \ldots, f_{n} ; \tau\right)$ with $f_{i} \in \mathcal{B}_{M}$ are linearly independent over $M_{*}$ by Theorem 3.1, as $M_{*} \subset \operatorname{Frac}\left(\mathrm{QM}_{*}\right)$.

A polynomial basis for $\mathcal{I}^{\mathrm{QM}}$. Recall from Proposition 2.3(ii) that $\mathrm{QM}_{*}$ is isomorphic to the polynomial algebra $\mathbb{C}\left[E_{2}, E_{4}, E_{6}\right]$. A similar, but slightly more involved statement holds for the $\mathrm{QM}_{*}$-algebra $\mathcal{I}^{\mathrm{QM}}$ of iterated integrals of quasimodular forms. Namely, $\mathcal{I}^{\mathrm{QM}}$ is a polynomial algebra over $\mathrm{QM}_{*}$ in infinitely many variables, which are given by certain Lyndon words.

In the following, if $(S,<)$ is a totally ordered set, we will endow the free monoid $S^{*}$ on $S$ with the lexicographical order induced by $<$. Also, the length of $w$ is simply the number of letters of $w$.

Definition 4.6. A Lyndon word on $S^{*}$ is a nontrivial word $w \in S^{*} \backslash\{1\}$ such that for all factorizations $w=u v$ with $u, v \neq 1$, we have $w<v$. We denote by $\operatorname{Lyn}\left(S^{*}\right)$ the set of all Lyndon words on $S^{*}$. 
Example 4.7. Let $S=\{a, b\}$ with total order $a<b$. Then the Lyndon words on $S^{*}$ of length at most four are

$$
a, b, a b, a a b, a b b, a a a b, a a b b, a b b b .
$$

Now for a field $k$ and any set $S$, define $k\langle S\rangle$ to be the shuffle algebra on the free $k$ vector space generated by $S$. If $k$ is of characteristic zero, then by the Milnor-Moore theorem [Milnor and Moore 1965], $k\langle S\rangle$ is isomorphic to a polynomial algebra (in possibly infinitely many variables). The following refinement is due to Radford.

Theorem 4.8 [Radford 1979]. If $k$ has characteristic zero, then $k\langle S\rangle$ is freely generated, as a $k$-algebra, by the set of Lyndon words $\operatorname{Lyn}\left(S^{*}\right)$. Equivalently, $k\langle S\rangle \cong k\left[\operatorname{Lyn}\left(S^{*}\right)\right]$, the polynomial algebra on $\operatorname{Lyn}\left(S^{*}\right)$.

Returning to quasimodular forms, consider again the $\mathbb{C}$-vector space

$$
V=\mathbb{C} \cdot E_{2} \oplus M_{*},
$$

and let $\mathcal{B}=\bigcup_{k \geq 0} \mathcal{B}_{k}$ be the homogeneous basis of $V$ given by $\mathcal{B}_{k}=\left\{E_{4}^{a} E_{6}^{b} \mid\right.$ $4 a+6 b=k\}$ for $k \neq 2$, and $\mathcal{B}_{2}=\left\{E_{2}\right\}$. The basis $\mathcal{B}$ can be ordered for the lexicographical order as follows: if $E_{4}^{a} E_{6}^{b}, E_{4}^{a^{\prime}} E_{6}^{b^{\prime}} \in \mathcal{B}_{k}$, then

$$
E_{4}^{a} E_{6}^{b}<E_{4}^{a^{\prime}} E_{6}^{b^{\prime}}: \Leftrightarrow a<a^{\prime} \text {, or } a=a^{\prime} \text { and } b<b^{\prime},
$$

and if $f \in \mathcal{B}_{k}, g \in \mathcal{B}_{k^{\prime}}$ with $k<k^{\prime}$, then $f<g$.

Now, since for $f_{1}, \ldots, f_{n} \in \mathcal{B}$, the iterated integrals $I\left(f_{1}, \ldots, f_{n} ; \tau\right)$ are linearly independent over $\mathrm{QM}_{*}$ (by Theorem 3.1), we can canonically identify the set of all $I\left(f_{1}, \ldots, f_{n} ; \tau\right)$ with the free monoid $\mathcal{B}^{*}$ and order $\mathcal{B}^{*}$ for the lexicographical ordering induced from the order on $\mathcal{B}$ above. The next result is a formal consequence of Theorems 4.3, 4.5 and 4.8.

Theorem 4.9. The elements of $\operatorname{Lyn}\left(\mathcal{B}^{*}\right)$ are algebraically independent over $\mathrm{QM}_{*}$ and we have a natural isomorphism of $\mathrm{QM}_{*}$-algebras

$$
\mathrm{QM}_{*}\left[\operatorname{Lyn}\left(\mathcal{B}^{*}\right)\right] \cong \mathcal{I}^{\mathrm{QM}},
$$

which is filtered for the length, where the left-hand side is the polynomial $\mathrm{QM}_{*}$ algebra on $\operatorname{Lyn}\left(\mathcal{B}^{*}\right)$. Explicitly, the isomorphism maps an element

$$
w=f_{1} \cdots f_{n} \in \operatorname{Lyn}\left(\mathcal{B}^{*}\right)
$$

to the iterated integral $I\left(f_{1}, \ldots, f_{n} ; \tau\right)$. Similarly, we have a natural isomorphism of $M_{*}$-algebras

$$
M_{*}\left[\operatorname{Lyn}\left(\mathcal{B}_{M}^{*}\right)\right] \cong \mathcal{I}^{M},
$$

where $\mathcal{B}_{M}=\mathcal{B} \backslash\left\{E_{2}\right\}$. 
Example 4.10. The following table gives all elements of $\operatorname{Lyn}\left(\mathcal{B}^{*}\right)$ involving iterated integrals of length at most two of quasimodular forms of total weight at most 12 . For ease of notation, we have dropped the $\tau$ from $I\left(f_{1}, \ldots, f_{n} ; \tau\right)$.

\begin{tabular}{|c|c|c|c|}
\cline { 2 - 4 } \multicolumn{1}{c|}{} & \multicolumn{2}{c|}{ Length } \\
\hline Weight & 0 & 1 & 2 \\
\hline 0 & - & $I(1)$ & - \\
2 & - & $I\left(E_{2}\right)$ & - \\
4 & - & $I\left(E_{4}\right)$ & $I\left(1, E_{4}\right)$ \\
6 & - & $I\left(E_{6}\right)$ & $I\left(1, E_{6}\right), I\left(E_{2}, E_{4}\right)$ \\
8 & - & $I\left(E_{4}^{2}\right)$ & $I\left(1, E_{4}^{2}\right), I\left(E_{2}, E_{6}\right)$ \\
10 & - & $I\left(E_{4} E_{6}\right)$ & $I\left(1, E_{4} E_{6}\right), I\left(E_{2}, E_{4}^{2}\right), I\left(E_{4}, E_{6}\right)$ \\
12 & - & $I\left(E_{4}^{3}\right), I\left(E_{6}^{2}\right)$ & $I\left(1, E_{4}^{3}\right), I\left(1, E_{6}^{2}\right), I\left(E_{2}, E_{4} E_{6}\right), I\left(E_{4}, E_{4}^{2}\right)$ \\
\hline
\end{tabular}

Also, the list of all elements of $\operatorname{Lyn}\left(\mathcal{B}^{*}\right)$ consisting of iterated integrals of length at most three of quasimodular forms of total weight 12 is given by

$$
\begin{aligned}
& \left\{I\left(E_{4}^{3}\right), I\left(E_{6}^{2}\right), I\left(1, E_{4}^{3}\right), I\left(1, E_{6}^{2}\right), I\left(E_{2}, E_{4} E_{6}\right), I\left(E_{4}, E_{4}^{2}\right),\right. \\
& I\left(1,1, E_{4}^{3}\right), I\left(1,1, E_{6}^{2}\right), I\left(1, E_{2}, E_{4} E_{6}\right), I\left(1, E_{4}, E_{4}^{2}\right), I\left(1, E_{6}, E_{6}\right), \\
& \left.I\left(1, E_{4}^{2}, E_{4}\right), I\left(1, E_{4} E_{6}, E_{2}\right), I\left(E_{2}, E_{2}, E_{4}^{2}\right), I\left(E_{2}, E_{4}, E_{6}\right), I\left(E_{2}, E_{6}, E_{4}\right)\right\} .
\end{aligned}
$$

\section{Appendix: Eichler-Shimura for quasimodular forms}

In this appendix, we show how one can attach one-cocycles to quasimodular forms. This extends the classical Eichler-Shimura theory of the cocycles attached to modular forms, and is probably well-known to the experts, but the author does not know of a suitable reference for the precise statements.

Throughout this appendix, we will freely use some elementary concepts from the cohomology of groups, for which we refer to [Weibel 1994, Chapter 6].

Cocycles attached to modular forms. We first briefly recall how modular forms give rise to cocycles for $\mathrm{SL}_{2}(\mathbb{Z})$. A standard reference is [Lang 1976, Chapter VI].

For $d \geq 0$, let $\mathbb{Q}[X, Y]_{d}$ be the $\mathbb{Q}$-vector space of homogeneous polynomials in $X$ and $Y$ of degree $d$. It is a right $\mathrm{SL}_{2}(\mathbb{Z})$-module by defining

$$
\left.P(X, Y)\right|_{\gamma}=P(a X+b Y, c X+d Y) \quad \text { for } \gamma=\left(\begin{array}{ll}
a & b \\
c & d
\end{array}\right) \in \mathrm{SL}_{2}(\mathbb{Z}), P \in \mathbb{Q}[X, Y]_{d} .
$$

With this action, given a modular form $f$ of weight $k \geq 2$, it is straightforward to verify that the holomorphic differential one-form

$$
\underline{f}(\tau):=(2 \pi i)^{k-1} f(\tau)(X-\tau Y)^{k-2} \mathrm{~d} \tau \in \Omega^{1}(\mathfrak{H}) \otimes_{\mathbb{Q}} \mathbb{Q}[X, Y]_{k-2}
$$


is $\mathrm{SL}_{2}(\mathbb{Z})$-invariant, where $\mathrm{SL}_{2}(\mathbb{Z})$ acts on $\mathfrak{H}$ in the usual way via fractional linear transformations. Fixing a base point $\tau_{0}$ of $\mathfrak{H}$ (possibly $i \infty$ ), it follows from the $\mathrm{SL}_{2}(\mathbb{Z})$-invariance that the function

$$
r_{f, \tau_{0}}: \mathrm{SL}_{2}(\mathbb{Z}) \rightarrow \mathbb{C}[X, Y]_{k-2}, \quad \gamma \mapsto \int_{\tau}^{\tau_{0}} \underline{f}(\tau)-\left.\left(\int_{\gamma \cdot \tau}^{\tau_{0}} \underline{f}(\tau)\right)\right|_{\gamma}
$$

(regularized as in Section 2 if $\tau_{0}=i \infty$ ) is a one-cocycle, i.e., it satisfies

$$
r_{f, \tau_{0}}\left(\gamma_{1} \gamma_{2}\right)=\left.r_{f, \tau_{0}}\left(\gamma_{1}\right)\right|_{\gamma_{2}}+r_{f, \tau_{0}}\left(\gamma_{2}\right)
$$

for all $\gamma_{1}, \gamma_{2} \in \mathrm{SL}_{2}(\mathbb{Z})$. Its cohomology class does not depend on $\tau_{0}$, and we denote this class simply by $\left[r_{f}\right]$.

The same construction can also be applied to the complex conjugate

$$
\underline{f(\tau)}:=(-2 \pi i)^{k-1} \overline{f(\tau)}(X-\bar{\tau} Y)^{k-2} \mathrm{~d} \bar{\tau}
$$

of the one-form $\bar{f}(\tau)$, and we denote by $\left[r_{\bar{f}}\right]$ the resulting cohomology class.

Theorem A.1 (Eichler-Shimura). For every $k \geq 2$, the morphism

$$
M_{k} \oplus \bar{S}_{k} \rightarrow H^{1}\left(\mathrm{SL}_{2}(\mathbb{Z}), \mathbb{Q}[X, Y]_{k-2}\right) \otimes_{\mathbb{Q}} \mathbb{C}, \quad(f, \bar{g}) \mapsto\left[r_{f}\right]+\left[r_{\bar{g}}\right]
$$

is an isomorphism of $\mathbb{C}$-vector spaces. Here, $\bar{S}_{k}$ denotes the complex conjugate of the $\mathbb{C}$-vector space of cusp forms of weight $k$.

Cocycles for the braid group. The fact that $r_{f}$ is a cocycle hinges on the modularity of $f$. In order to incorporate quasimodular forms into the picture, we need to consider instead of $\mathrm{SL}_{2}(\mathbb{Z})$ the braid group $B_{3}=\left\langle\sigma_{1}, \sigma_{2}: \sigma_{1} \sigma_{2} \sigma_{1}=\sigma_{2} \sigma_{1} \sigma_{2}\right\rangle$ on three strands. It is a central extension

$$
1 \rightarrow \mathbb{Z} \rightarrow B_{3} \rightarrow \mathrm{SL}_{2}(\mathbb{Z}) \rightarrow 1,
$$

and also the fundamental group of the quotient of $\mathbb{C}^{\times} \times \mathfrak{H}$ by the $\mathrm{SL}_{2}(\mathbb{Z})$-action

$$
\gamma \cdot(z, \tau)=((c \tau+d) z, \gamma \cdot \tau) \quad \text { for } \gamma=\left(\begin{array}{ll}
a & b \\
c & d
\end{array}\right) \in \mathrm{SL}_{2}(\mathbb{Z}),
$$

where $\mathrm{SL}_{2}(\mathbb{Z})$ acts on $\mathfrak{H}$ as before. We refer to [Hain 2011, §8] for more details and further equivalent descriptions of $B_{3}$.

Next, we compute the cohomology groups $H^{1}\left(B_{3}, \mathbb{Q}[X, Y]_{d}\right)$, where $B_{3}$ acts on $\mathbb{Q}[X, Y]_{d}$ via the projection $B_{3} \rightarrow \mathrm{SL}_{2}(\mathbb{Z})$.

Proposition A.2. We have canonical isomorphisms

$$
H^{1}\left(B_{3}, \mathbb{Q}[X, Y]_{d}\right) \cong \begin{cases}H^{1}\left(\mathrm{SL}_{2}(\mathbb{Z}), \mathbb{Q}[X, Y]_{d}\right) & \text { for } d \geq 1, \\ \mathbb{Q} & \text { for } d=0 .\end{cases}
$$


Proof. The Hochschild-Serre spectral sequence (see [Weibel 1994, §6.8.3]) associated to the extension (A-1) yields an exact sequence

$0 \rightarrow H^{1}\left(\mathrm{SL}_{2}(\mathbb{Z}), \mathbb{Q}[X, Y]_{d}\right) \rightarrow H^{1}\left(B_{3}, \mathbb{Q}[X, Y]_{d}\right) \rightarrow H^{1}\left(\mathbb{Z}, \mathbb{Q}[X, Y]_{d}\right)^{\mathrm{SL}_{2}(\mathbb{Z})} \rightarrow 0$,

where we have used the fact that $H^{2}\left(\mathrm{SL}_{2}(\mathbb{Z}), \mathbb{Q}[X, Y]_{d}\right)=\{0\}$, as $\mathrm{SL}_{2}(\mathbb{Z})$ has virtual cohomological dimension equal to one. The proposition now follows easily from this.

Quasimodular forms and braid group cocycles. In light of Theorem A.1, the isomorphisms of Proposition A.2 suggest attaching a one-cocycle $B_{3} \rightarrow \mathbb{C}$ to the Eisenstein series $E_{2}$. Indeed, this can be done as follows.

First, the modular transformation property of $E_{2}$ (2-2) implies that the differential one-form

$$
2 \pi i E_{2}(\tau) \mathrm{d} \tau-12 \frac{\mathrm{d} z}{z} \in \Omega^{1}\left(\mathbb{C}^{\times} \times \mathfrak{H}\right)
$$

is $\mathrm{SL}_{2}(\mathbb{Z})$-invariant, i.e., it descends to the quotient $\mathrm{SL}_{2}(\mathbb{Z}) \backslash\left(\mathbb{C}^{\times} \times \mathfrak{H}\right)$. Denote by

$$
\underline{E_{2}}(\xi, \tau):=\varphi^{*}\left(2 \pi i E_{2}(\tau) \mathrm{d} \tau-12 \frac{\mathrm{d} z}{z}\right)=2 \pi i E_{2}(\tau) \mathrm{d} \tau-12 \mathrm{~d} \xi \in \Omega^{1}(\mathbb{C} \times \mathfrak{H})
$$

the pull-back of (A-2) along the universal covering map $\varphi: \mathbb{C} \times \mathfrak{H} \rightarrow \mathrm{SL}_{2}(\mathbb{Z}) \backslash\left(\mathbb{C}^{\times} \times \mathfrak{H}\right)$. Clearly, $E_{2}(\xi, \tau)$ is $B_{3}$-invariant and it follows that for any base point $\left(\xi_{0}, \tau_{0}\right)$ (for example, $\left.\left(\xi_{0}, \tau_{0}\right)=(0, i \infty)\right)$, the function

$$
r_{E_{2},\left(\xi_{0}, \tau_{0}\right)}: B_{3} \rightarrow \mathbb{C}, \quad \gamma \mapsto \int_{(\xi, \tau)}^{\left(\xi_{0}, \tau_{0}\right)} \underline{E_{2}}(\xi, \tau)-\left.\left(\int_{\gamma \cdot(\xi, \tau)}^{\left(\xi_{0}, \tau_{0}\right)} \underline{E_{2}}(\xi, \tau)\right)\right|_{\gamma}
$$

is a well-defined cocycle (again, regularization is needed if $\tau_{0}=i \infty$ ).

Remark A.3. The integral $I\left(E_{2} ; \tau\right)$ introduced in Section 2 is actually equal to $\int_{\tau}^{i \infty} \underline{E_{2}}(\xi, \tau)$, where we embed $\mathfrak{H}$ into $\mathbb{C} \times \mathfrak{H}$ by $\tau \mapsto(0, \tau)$. However, that embedding is not $B_{3}$-equivariant, and indeed the integral $I\left(E_{2} ; \tau\right)$ does not give rise to a cocycle for $B_{3}$; for this, one really needs to lift the form $2 \pi i E_{2}(\tau) \mathrm{d} \tau$ to the form $\underline{E_{2}}(\xi, \tau)$.

Now, since the cocycle $r_{E_{2},\left(\xi_{0}, \tau_{0}\right)}$ is nonzero, its cohomology class (which is again independent of the choice of base point $\left.\left(\xi_{0}, \tau_{0}\right)\right)$ is nontrivial. The EichlerShimura theorem (Theorem A.1) together with Proposition A.2 then implies the next result.

Corollary A.4. For every $k \geq 2$, the morphism

$$
V_{k} \oplus \bar{S}_{k} \rightarrow H^{1}\left(B_{3}, \mathbb{Q}[X, Y]_{k-2}\right) \otimes_{\mathbb{Q}} \mathbb{C}, \quad(f, \bar{g}) \mapsto\left[r_{f}\right]+\left[r_{\bar{g}}\right],
$$

where $V:=M_{*} \oplus \mathbb{C} \cdot E_{2}$, is an isomorphism of $\mathbb{C}$-vector spaces. 
One can also attach a cocycle $r_{f, \tau_{0}}$ to a general quasimodular form $f \in \mathrm{QM}_{k}$ of weight $k$ as follows. By Proposition 2.3(ii), we know that $f$ can be written uniquely as a $\mathbb{C}$-linear combination of derivatives of modular forms and of derivatives of $E_{2}$. Thus, we can write

$$
f=\sum \lambda_{g} \cdot D^{p_{g}}(g), \quad \lambda_{g} \in \mathbb{C}, p_{g} \geq 0,
$$

where either $g$ is a modular form of weight $k-2 p_{g}$ or $g=E_{2}$. Therefore, we may define $r_{f, \tau_{0}}: B_{3} \rightarrow \mathbb{C}[X, Y]_{\leq k-2}:=\bigoplus_{0 \leq d \leq k-2} \mathbb{C}[X, Y]_{d}$ by

$$
r_{f, \tau_{0}}:=\sum \lambda_{g} \cdot r_{g, \tau_{0}} \text {. }
$$

Using this definition, one sees in particular that the cocycles of quasimodular forms can be expressed in terms of the cocycles attached to modular forms and to $E_{2}$. This is of course in line with Corollary A.4.

Remark A.5. In [Manin 2006; Brown 2016; Hain 2016], certain nonabelian $\mathrm{SL}_{2}(\mathbb{Z})$-cocycles given in terms of iterated integrals of modular forms are studied. It would be natural to try and extend this theory to nonabelian $B_{3}$-cocycles attached to iterated integrals of quasimodular forms (perhaps along the lines suggested in [Hain 2016, §14]), but this is beyond the scope of the present paper.

\section{Acknowledgments}

Very many thanks to Pierre Lochak for bringing the article [Deneufchâtel et al. 2011] to the author's attention. Also, many thanks to Francis Brown, Erik Panzer and the referees for corrections as well as very helpful suggestions and to Don Zagier for inspiring discussions on the Appendix. The results of this paper were found while the author was a Ph.D. student at Universität Hamburg under the supervision of Ulf Kühn.

\section{References}

[Bloch and Okounkov 2000] S. Bloch and A. Okounkov, "The character of the infinite wedge representation", Adv. Math. 149:1 (2000), 1-60. MR Zbl

[Brown 2009] F. C. S. Brown, "Multiple zeta values and periods of moduli spaces $\overline{\mathfrak{M}}_{0, n}$ ", Ann. Sci. Éc. Norm. Supér. (4) 42:3 (2009), 371-489. MR Zbl

[Brown 2016] F. Brown, "Multiple modular values and the relative completion of the fundamental group of $M_{1,1}$ ", preprint, 2016. arXiv

[Brown 2017] F. Brown, "A class of non-holomorphic modular forms, II: Equivariant iterated Eisenstein integrals", preprint, 2017. arXiv

[Deneufchâtel et al. 2011] M. Deneufchâtel, G. H. E. Duchamp, V. H. N. Minh, and A. I. Solomon, "Independence of hyperlogarithms over function fields via algebraic combinatorics", pp. 127-139 in Algebraic informatics (Linz, Austria, 2011), edited by F. Winkler, Lecture Notes in Comput. Sci. 6742, Springer, 2011. MR Zbl 
[Eichler 1957] M. Eichler, "Eine Verallgemeinerung der Abelschen Integrale”, Math. Z. 67 (1957), 267-298. MR Zbl

[Hain 1987] R. M. Hain, "The geometry of the mixed Hodge structure on the fundamental group", pp. 247-282 in Algebraic geometry (Brunswick, ME, 1985), edited by S. J. Bloch, Proceedings of Symposia in Pure Math. 46, American Mathematical Society, Providence, RI, 1987. MR Zbl

[Hain 2011] R. Hain, "Lectures on moduli spaces of elliptic curves", pp. 95-166 in Transformation groups and moduli spaces of curves (Hangzhou, China), edited by L. Ji and S.-T. Yau, Advanced Lectures in Math. 16, International Press, Somerville, MA, 2011. MR Zbl

[Hain 2016] R. Hain, "The Hodge-de Rham theory of modular groups", pp. 422-514 in Recent advances in Hodge theory (Vancouver, 2013), edited by M. Kerr and G. Pearlstein, London Math. Society Lecture Note Series 427, Cambridge University Press, 2016. MR Zbl

[Kaneko and Zagier 1995] M. Kaneko and D. Zagier, "A generalized Jacobi theta function and quasimodular forms", pp. 165-172 in The moduli space of curves (Texel, Netherlands, 1994), edited by R. Dijkgraaf et al., Progress in Math. 129, Birkhäuser, Boston, 1995. MR Zbl

[Lang 1976] S. Lang, Introduction to modular forms, Grundlehren der math. Wissenschaften 222, Springer, 1976. MR Zbl

[Lochak et al. 2017] P. Lochak, N. Matthes, and L. Schneps, "Elliptic multiple zeta values and the elliptic double shuffle relations", preprint, 2017. arXiv

[Manin 2006] Y. I. Manin, "Iterated integrals of modular forms and noncommutative modular symbols", pp. 565-597 in Algebraic geometry and number theory, edited by V. Ginzburg, Progress in Math. 253, Birkhäuser, Boston, 2006. MR Zbl

[Martin and Royer 2005] F. Martin and E. Royer, "Formes modulaires et périodes", pp. 1-117 in Formes modulaires et transcendance (Marseille, 2003), edited by S. Fischler et al., Sémin. Congr. 12, Société Mathématique de France, Paris, 2005. MR Zbl

[Milnor and Moore 1965] J. W. Milnor and J. C. Moore, "On the structure of Hopf algebras", Ann. of Math. (2) 81 (1965), 211-264. MR Zbl

[Radford 1979] D. E. Radford, "A natural ring basis for the shuffle algebra and an application to group schemes”, J. Algebra 58:2 (1979), 432-454. MR Zbl

[Reutenauer 1993] C. Reutenauer, Free Lie algebras, London Math. Society Monographs New Series 7, Oxford University Press, 1993. MR Zbl

[Weibel 1994] C. A. Weibel, An introduction to homological algebra, Cambridge Studies in Advanced Math. 38, Cambridge University Press, 1994. MR Zbl

[Zagier 2008] D. Zagier, "Elliptic modular forms and their applications", pp. 1-103 in The 1-2-3 of modular forms (Nordfjordeid, Norway, 2004), edited by K. Ranestad, Springer, 2008. MR Zbl

Communicated by Yuri Manin

Received 2016-11-08 Revised 2017-06-15 Accepted 2017-09-08

nilsmath@mpim-bonn.mpg.de Max-Planck-Institut für Mathematik, Bonn, Germany 


\section{Algebra \& Number Theory}

msp.org/ant

\section{EDITORS}

MANAGING EDITOR

Bjorn Poonen

Massachusetts Institute of Technology

Cambridge, USA

\author{
EDITORIAL BOARD CHAIR \\ David Eisenbud \\ University of California \\ Berkeley, USA
}

BOARD OF EDITORS

Richard E. Borcherds

J-L. Colliot-Thélène

Brian D. Conrad

Samit Dasgupta

Hélène Esnault

Gavril Farkas

Hubert Flenner

Sergey Fomin

Edward Frenkel

Andrew Granville

Joseph Gubeladze

Roger Heath-Brown

Craig Huneke

Kiran S. Kedlaya

János Kollár

Yuri Manin

Philippe Michel

Susan Montgomery

Shigefumi Mori
University of California, Berkeley, USA

CNRS, Université Paris-Sud, France

Stanford University, USA

University of California, Santa Cruz, USA

Freie Universität Berlin, Germany

Humboldt Universität zu Berlin, Germany

Ruhr-Universität, Germany

University of Michigan, USA

University of California, Berkeley, USA

Université de Montréal, Canada

San Francisco State University, USA

Oxford University, UK

University of Virginia, USA

Univ. of California, San Diego, USA

Princeton University, USA

Northwestern University, USA

École Polytechnique Fédérale de Lausanne

University of Southern California, USA

RIMS, Kyoto University, Japan
Martin Olsson

Raman Parimala

Jonathan Pila

Anand Pillay

Michael Rapoport

Victor Reiner

Peter Sarnak

Joseph H. Silverman

Michael Singer

Christopher Skinner

Vasudevan Srinivas

J. Toby Stafford

Pham Huu Tiep

Ravi Vakil

Michel van den Bergh

Marie-France Vignéras

Kei-Ichi Watanabe

Shou-Wu Zhang
University of California, Berkeley, USA

Emory University, USA

University of Oxford, UK

University of Notre Dame, USA

Universität Bonn, Germany

University of Minnesota, USA

Princeton University, USA

Brown University, USA

North Carolina State University, USA

Princeton University, USA

Tata Inst. of Fund. Research, India

University of Michigan, USA

University of Arizona, USA

Stanford University, USA

Hasselt University, Belgium

Université Paris VII, France

Nihon University, Japan

Princeton University, USA

PRODUCTION

production@msp.org

Silvio Levy, Scientific Editor

See inside back cover or msp.org/ant for submission instructions.

The subscription price for 2017 is US $\$ 325 /$ year for the electronic version, and $\$ 520 /$ year ( $\$ 55$, if shipping outside the US) for print and electronic. Subscriptions, requests for back issues and changes of subscriber address should be sent to MSP.

Algebra \& Number Theory (ISSN 1944-7833 electronic, 1937-0652 printed) at Mathematical Sciences Publishers, 798 Evans Hall \#3840, c/o University of California, Berkeley, CA 94720-3840 is published continuously online. Periodical rate postage paid at Berkeley, CA 94704, and additional mailing offices.

ANT peer review and production are managed by EditFLOW ${ }^{\circledR}$ from MSP.

\section{PUBLISHED BY}

mathematical sciences publishers

nonprofit scientific publishing

http://msp.org/

(C) 2017 Mathematical Sciences Publishers 


\section{Algebra \& Number Theory}

Volume $11 \quad$ No. $9 \quad 2017$

A nonarchimedean Ax-Lindemann theorem

ANTOINE CHAMBERT-LOIR and FRANÇOIS LOESER

A modular description of $\mathscr{X}_{0}(n)$

KĘSTUTIS ČESNAVIČIUS

Elementary equivalence versus isomorphism, II

FLORIAN POP

On the algebraic structure of iterated integrals of quasimodular forms

NiLS MATTHES

On the density of zeros of linear combinations of Euler products for $\sigma>1$

MATtia RighetTi

Adams operations on matrix factorizations

Michael K. Brown, Claudia Miller, Peder Thompson and

MARK E. WALKER

Rationality does not specialize among terminal fourfolds

ALEXANDER PERRY

Topological noetherianity for cubic polynomials

HARM DERKSEN, Rob H. EgGERMONT and ANDREW SNOWDEN 